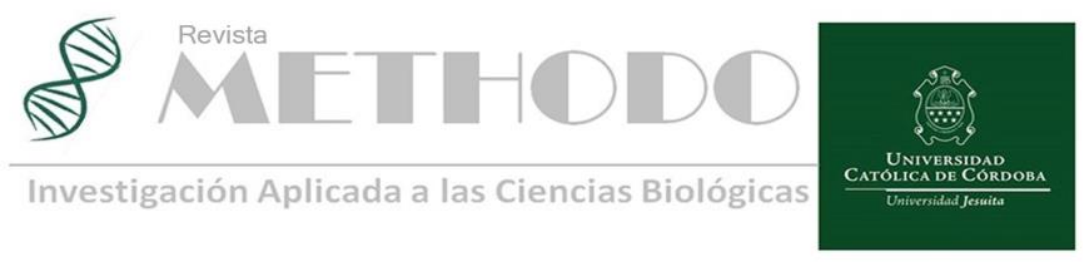

\title{
Sars-cov-2 y sus efectos en el primer trimestre en Argentina
}

\section{Sars-cov-2 and its effects in the first quarter in Argentina}

\author{
Tania Terán ${ }^{1}$ (D), Andrea Layedra ${ }^{1}$, Luis A. Martinez ${ }^{1}$, María Fernanda Cadena ${ }^{1}$, Maximiliano S. Pérez ${ }^{1,2}$, \\ Betiana Lerner ${ }^{1,2}$ (iD. \\ 1 Universidad Tecnológica Nacional (UTN), Facultad Regional Haedo, Provincia de Buenos Aires. \\ 2 Universidad de Buenos Aires (UBA), Instituto de Ingeniería, Ciudad de Buenos Aires. \\ Correspondencia: Maximiliano S. Pérez. Universidad Tecnológica Nacional (UTN), Facultad Regional Haedo, Provincia de Buenos Aires CP 1706, \\ Universidad de Buenos Aires (UBA) Instituto de Ingeniería, Ciudad de Buenos Aires. Email: mperez@ frh.utn.edu.ar. \\ Betania Lerner. Universidad Tecnológica Nacional (UTN), Facultad Regional Haedo, Provincia de Buenos Aires CP 1706, Universidad de Buenos Aires \\ (UBA) Instituto de Ingeniería, Ciudad de Buenos Aires. Email: blerner@frh.utn.edu.ar.
}

\section{Resumen}

INTRODUCCIÓN: El nuevo virus SARS-CoV-2 es causante de una enfermedad llamada COVID-19 que puede tornarse grave e incluso mortal. El número de casos y fallecidos reportados y el impacto a nivel socioeconómico dan una idea de la gravedad a nivel global que ha generado el mismo. Al ser un nuevo virus, se desconoce aún varios aspectos de este, por lo que el objetivo de este artículo es resumir los principales descubrimientos en torno a este nuevo virus y analizar los efectos de este en Argentina durante el primer trimestre del año en curso, con énfasis en la tasa de infección, la tasa de mortalidad y los casos recuperados para evaluar si las medidas de aislamiento y protección han sido efectivas.

DESARROLLO: Para esto se graficó el número de infectados, número de recuperados y número de fallecidos bajo diferentes enfoques, además de realizar una revisión de la literatura sobre su origen, mecanismo de infección, síntomas, métodos de diagnóstico, tratamiento, vacuna y medidas de atenuación. CONCLUSIÓN: Se concluye que aún quedan muchos vacíos que llenar sobre información de este virus para poder aplicar una solución farmacológica, mientras tanto la recomendación por parte de la Organización Mundial de la Salud y el Ministerio de Salud Argentino es mantener el distanciamiento social y el uso obligatorio de mascarillas.

Palabras claves: SARS-CoV-2, COVID-19, cuarentena, mortalidad, letalidad.

\begin{abstract}
Introduction: The new SARS-CoV-2 virus causes a disease called COVID-19 that can become serious and even fatal. The number of cases and deaths reported and the impact at the socioeconomic level give an idea of the global severity that it has generated. As it is a new virus, several aspects of it are still unknown, so the objective of this article is to summarize the main discoveries around this new virus and analyze its effects in Argentina during the first quarter of this year. with an emphasis on the infection rate, the mortality rate, and the cases recovered to evaluate whether the isolation and protection measures have been effective. Development: For this, the number of infected, number of recovered, and number of deceased were plotted under different approaches, in addition to conducting a review of the literature on its origin, infection mechanism, symptoms, diagnostic methods, treatment, vaccine, and measures of attenuation. Conclusion:
\end{abstract}

Revista Methodo: Investigación Aplicada a las Ciencias Biológicas. Universidad Católica de Córdoba. Jacinto Ríos 571 Bo Gral. Paz. X5004FXS. Córdoba. Argentina. Tel.: (54) 3514517299 / Correo: methodo@ucc.edu.ar / Web: methodo.ucc.edu.ar | ARTICULO DE REVISION Rev. Methodo 2021;6(1):20-32. 
It is concluded that there are still many gaps to fill regarding the information on this virus to apply a pharmacological solution, meanwhile the recommendation by the World Health Organization and the Argentine Ministry of Health is to maintain social distancing and the mandatory use of masks.

Key words: SARS-CoV-2, COVID-19, quarantine, mortality, lethality.

\section{Introducción}

Los coronavirus pertenecen a la familia Coronaviridae en el orden Nidovirales, poseen ARN con envoltura con más de doce patógenos específicos de aves y mamíferos. Hay un total de siete coronavirus que se sabe que infectan a los humanos; HKU1, NL63, OC43 y 229E que están asociados con síntomas leves y SARS-CoV, MERS-CoV y el virus que causa la nueva pandemia de neumonía (COVID-19) SARS-CoV2, que puede causar una enfermedad grave $\mathrm{e}^{1,2,3}$.

Los efectos del virus SARS-CoV-2 desde que inició la pandemia en Wuhan - China, se han evidenciado en todo el mundo. El número de casos, fallecidos reportados y el impacto a nivel socioeconómico dan una idea de la gravedad a nivel global que ha generado el mismo. Al ser un nuevo virus, se desconoce aún varios aspectos de este, por lo que los esfuerzos del mundo científico se han concentrado en revelar todas sus características, dando lugar a una gran cantidad de publicaciones científicas.

Por lo tanto, el objetivo de este artículo es resumir los principales descubrimientos en torno a este nuevo virus valorando la literatura actual y analizar los efectos de este en Argentina durante el primer trimestre del año en curso, con énfasis en la tasa de infección, la tasa de mortalidad y los casos recuperados para evaluar si las medidas de aislamiento y protección han sido efectivas.

\section{Desarrollo}

Al igual que todos los coronavirus, SARS-CoV-2 posee picos en forma de corona en la superficie exterior. Estos picos son llamados proteínas espigas $(S)$, parte más variable del genoma del coronavirus, las cuales van a unirse al receptor de la enzima convertidora de la angiotensina 2 (ACE2) de la célula del huésped ${ }^{4}$.

El virus ingresa principalmente por las vías aéreas, por lo que tiene mayor facilidad de unión a los receptores ACE2 de las células epiteliales alveolares. Esto activa las células inmunes e induce la secreción de citoquinas y quimiocinas inflamatorias en las células endoteliales vasculares pulmonares ${ }^{4}$.

\section{Origen}

La optimización que el SARS-CoV-2 tiene para unirse con alta afinidad al receptor ACE2 de los humanos proviene del dominio de unión al receptor (RBD), un segmento en la proteína espiga. A través del análisis computacional, se predijo que la interacción entre ACE2 y el RBD no es ideal. La secuencia RBD es diferente de las mostradas en SARS-CoV para ser óptima la unión al receptor. Por lo tanto, el SARS-CoV-2 no es el producto de la manipulación, lo más probable es que sea el resultado de una selección natural en un humano o ACE2 de tipo humano que permite que surja otra solución de unión óptima ${ }^{5,6,7,8,9}$.

Algunas teorías explican el origen natural del SARS-CoV-2. Además, si se hubiera realizado la manipulación genética, probablemente se habría utilizado uno de los varios sistemas de genética inversa de cualquier red troncal de virus utilizada anteriormente, por lo que el origen de este nuevo virus es altamente improbable que se haya realizado mediante la manipulación de laboratorio de un coronavirus relacionado con el SARS-CoV ${ }^{5}$. Algunos coronavirus de pangolín exhiben una fuerte similitud con el SARS-CoV-2 en el RBD, lo que enfatiza la teoría de que la proteína de la punta del SARS-CoV-2 optimizada para unirse a ACE2 de tipo humano es el resultado de la selección natural. Además, pueden ocurrir mutaciones, inserciones y deleciones cerca de la unión S1-S2 de los coronavirus, lo que demuestra que el sitio de escisión polibásica puede surgir por un proceso evolutivo natural. Todavía falta un gen que codifique ACE2 que sea similar al ortólogo humano $5,10,11,12,13$.

Basado en las similitudes entre la RBD de los coronavirus de pangolín y el SARS-CoV-2, también es probable que el virus que saltó al ser humano mute la inserción del sitio de escisión polibásica durante la transmisión de humano a humano a través de la adaptación. En este caso, debería haberse producido un período de transmisión no reconocida en humanos entre el evento zoonótico inicial ${ }^{5,10,11,12,13 .}$

Se encontró evidencia genómica y evolutiva de la aparición de un coronavirus similar al SARS-CoV2 (llamado Pangolin-CoV) en pangolines malayos muertos. Pangolin-CoV es $91.02 \%$ y $90.55 \%$ idéntico a SARS-CoV-2 y BatCoV RaTG13 
(coronavirus de murciélago) respectivamente, a nivel de genoma completo. Cinco aminoácidos clave en el RBD son consistentes entre el Pangolin-CoV y el SARS-CoV-2, colocándolo como el segundo pariente más cercano detrás de RaTG13. Por lo que se sugiere que las especies de pangolín son un reservorio natural de coronavirus similares al SARS-CoV-210,11, 12, 13.

En todas las filogenias, Pangolin-CoV, RaTG13 y SARS-CoV-2 se congregaron en un grupo bien respaldado, denominado el "grupo SARS-CoV-2". Este grupo representa un nuevo grupo de Betacoronavirus. Dentro de este grupo, RaTG13 y SARS-CoV-2 se agruparon juntos, y PangolinCoV fue su ancestro común más cercano ${ }^{10,11,12,13 .}$

\section{Infección}

El patrón de desprendimiento del ácido nucleico viral de pacientes infectados se parece al de pacientes con influenza, existiendo potencial transmisión en pacientes asintomáticos o poco sintomáticos por lo que la transmisión puede ocurrir de forma temprana en el curso de la infección ${ }^{14}$. Estudios muestran que el número de reproducción básico estimado (R0) es de 2.2, lo que indica que, en promedio cada persona infectada propaga la infección a dos personas adicionales; hasta que este número caiga por debajo de 1.0 es probable que el brote continúe extendiéndose ${ }^{15}$. Informes recientes de altos títulos de virus en la orofaringe al comienzo de la enfermedad, despiertan preocupación sobre el aumento de la infectividad durante el periodo de síntomas mínimos ${ }^{16}$.

Los fómites son la fuente principal de partículas infecciosas y está demostrado que otros coronavirus pueden permanecer hasta 10 días en superficies sin limpiar. Además, se detectó ARN del virus SARSCov-2 en muestras de heces de pacientes ${ }^{4}$.

Se realizó un estudio para estimar la duración del período de incubación de COVID-19, con 181 casos confirmados con exposición identificable y casos de inicio de síntomas informados entre el 4 de enero de 2020 y el 24 de febrero de 2020 fuera de la provincia de Hubei, China. La media del período de incubación fue de 5.1 días (IC 95\%, 4.5 a 5.8 días), y el $97.5 \%$ de aquellos que desarrollan síntomas dentro de 11.5 días (IC, 8.2 a 15.6 días) de infección. Estas estimaciones implican que 101 de cada 10000 casos desarrollarán síntomas después de 14 días de monitoreo activo o cuarentena ${ }^{17}$.

Aunque SARS-CoV-2, es filogenéticamente similar al Síndrome Respiratorio Agudo Severo (SARS-CoV) en 2003 y al Síndrome Respiratorio de Medio Oriente (MERS-CoV) en 2012, sus características de enfermedad como la tasa de reproducción (R0), la tasa de letalidad (CFR) y la sintomatología se parece más al virus de la gripe estacional. Sin embargo, SARS- CoV-2 parece ser más contagioso y tener un CFR 2 veces mayor que la influenza estacional ${ }^{18}$.

\section{Síntomas}

Los primeros pacientes confirmados como COVID-19 positivos (41) fueron hospitalizados el 16 de diciembre de 2019. Los pacientes con enfermedad grave desarrollaron SDRA (Síndrome de dificultad respiratoria aguda) en solo dos días. La condición clínica más común fue la neumonía grave, a veces mortal ${ }^{2}$.

Basado en 50,466 casos de COVID-19, un metaanálisis de pacientes hospitalizados mostró que el síndrome de dificultad respiratoria aguda (SDRA) ocurrió en el $14.8 \%$ de los pacientes con COVID-19, que era menos del $20 \%$ de los pacientes con síndrome respiratorio agudo severo (SARS); los sobrevivientes del síndrome de dificultad respiratoria aguda (SDRA) a menudo desarrollan fibrosis pulmonar, mientras que el $36 \%$ y el $30 \%$ de los pacientes con síndrome respiratorio agudo severo (SRAS) desarrollan, respectivamente, a los 3 y 6 meses después de la infección, fibrosis pulmonar. ${ }^{19}$

Los síntomas comunes que presentaron los pacientes fueron: fiebre, tos seca, disnea $\mathrm{y}$ opacidades bilaterales de vidrio esmerilado en las tomografías computarizadas de tórax. Esto dificulta mucho la diferenciación de la influenza, el virus sincitial respiratorio y otros virus respiratorios que son altamente prevalentes durante esta época del año ${ }^{1,2}$. En diciembre, son frecuentes estos virus en China.

COVID-19 desencadena una fuerte respuesta inmune inflamatoria innata, mayor secreción de citocinas T-helper-2 (Th2) (p. Ej., IL4 e IL10) que suprimen la inflamación y el agotamiento de los linfocitos después de la infección. Por lo tanto, posiblemente el exceso de inmunidad del huésped puede estar asociado con la patogénesis de COVID-19, $19^{1,2}$.

Se han destacado los pulmones como el principal órgano involucrado en la enfermedad, sin embargo, han informado que el SARS-CoV-2 está involucrado en el daño de otros órganos, como el hígado y los riñones, lo que afecta el metabolismo y la excreción de los medicamentos tomados para tratar la enfermedad ${ }^{20}$.

La anormalidad de la función hepática, el daño hepático agudo, como se observa en el SARS o la influenza grave, y el daño a múltiples tejidos u órganos además de la lesión hepática también estuvieron presentes en pacientes con COVID-192. La incidencia de anomalías hepáticas aumenta significativamente después de la infección con

Revista Methodo: Investigación Aplicada a las Ciencias Biológicas. Universidad Católica de Córdoba. Jacinto 
COVID-19 y durante el curso de la enfermedad, lo que puede indicar el efecto del SARS-CoV-2 en el hígado o los efectos secundarios de los medicamentos utilizados por los pacientes ${ }^{13}$. Además de las lesiones hepáticas, algunos artículos también informaron una mayor incidencia de lesión renal aguda después de COVID-19, que podría deberse a la presencia de SARS-CoV-2, la inflamación inducida por la enfermedad o un efecto sinérgico de ambos en riñones $^{21}$. Por lo tanto, el hígado y los riñones pueden dañarse en pacientes con COVID-19, lo que puede dificultar alcanzar la dosis terapéutica de los medicamentos y aumentar el riesgo de reacciones adversas a los medicamentos en los pacientes $^{20}$.

Los pacientes también pueden presentar síntomas gastrointestinales como náuseas o diarrea. Curiosamente, el receptor de entrada celular ACE2 parece mediar en la entrada de SARS-CoV-2 (similar al SARS) y se ha demostrado que se expresa altamente en los enterocitos del intestino delgado. ACE2 es importante para controlar la inflamación intestinal y su interrupción puede provocar diarrea. La frecuencia reportada de diarrea entre los pacientes con COVID-19 ha variado del 2 al 33\%, fue uno de los síntomas prominentes reportados por el primer caso en los Estados Unidos ya que el SARS-CoV-2 se detectó en las heces de los pacientes con COVID-19. Por lo tanto, el tracto gastrointestinal puede ser otra ruta potencial de infección. Algunos de los hallazgos de laboratorio más comunes descritos en pacientes con COVID-19 incluyen anormalidades en las pruebas de función hepática, leucopenia (reportada en $9-25 \%$ de los casos) o leucocitosis (24-30\%), alanina aminotransferasa elevada y aspartato aminotransferasa se han visto en hasta al $37 \%$ de los casos. Descripciones más recientes de pacientes en China también señalaron que alrededor del $10 \%$ de los pacientes también tenían niveles elevados de bilirrubina total ${ }^{22}$.

\section{Métodos de Diagnóstico}

Hay tres formas principales de detectar SARSCoV-2 en muestras respiratorias, sanguíneas y fecales, cada una con su valor de diagnóstico distinto: ${ }^{18}$

- Eliminación activa del virus: reacción en cadena de la polimerasa con transcripción inversa (RTPCR) del ARN monocatenario SARS-CoV-2. El tiempo de espera para la prueba puede ser tan corto como 3-6 horas, pero la RT-PCR requiere máquinas especializadas, kits de prueba $\mathrm{y}$ experiencia, por lo que el tiempo para obtener un resultado es mayor ${ }^{18}$.

- Con presencia de síntomas y signos de neumonía en entornos hospitalarios con alta carga de SARS-
CoV-2 y donde el muestreo es un riesgo demasiado alto [(por ejemplo, escasez de equipo de protección personal (EPP)]: tomografía computarizada de tórax con sus características de opacidades de vidrio esmerilado y la consolidación puede ser útil $^{18}$.

- Infección pasada: las pruebas serológicas ahora están disponibles, pero las tasas de falsos positivos y falsos negativos aún son inciertas ${ }^{18}$.

\section{Tipos de tratamientos y eficiencia}

El tratamiento del SARS implicó la terapia combinada de lopinavir y ritonavir y se asoció con un beneficio clínico sustancial con menos resultados clínicos adversos. Un profármaco de nucleótidos antivirales de amplio espectro llamado remdesivir presentó una potente eficacia para el tratamiento del coronavirus MERS y el coronavirus SARS en estudios preclínicos ${ }^{1}$.

Lopinavir y ritonavir fueron los primeros medicamentos que se utilizaron como tratamiento para COVID-19. Además, se administraron empíricamente antibióticos de temporada de influenza (por vía oral e intravenosa) y oseltamivir (por vía oral $75 \mathrm{mg}$ dos veces al día). Cuando la neumonía estaba presente, se aplicó la terapia con corticosteroides. Soporte de oxígeno para la hipoxemia ${ }^{4}$.

Para los pacientes que se han complicado, el tratamiento de apoyo incluye terapia de reemplazo renal continuo (TRRC), ventilación mecánica invasiva, oxigenación por membrana intracorpórea (ECMO). No se ha confirmado la efectividad de medicamentos antivirales específicos. Se ha sugerido el fármaco Barictinib como un potencial tratamiento para reducir el proceso de invasión e inflamación de virus ${ }^{4}$.

El fosfato de cloroquina puede inhibir la exacerbación de la neumonía, mejorar los hallazgos de las imágenes pulmonares, promover una conversión negativa del virus y acortar el curso de la enfermedad ${ }^{23}$. Se ha demostrado que la cloroquina/hidroxicloroquina, un medicamento de primera línea utilizado en el tratamiento y la profilaxis de la malaria, también utilizada en enfermedades autoinmunes, inhibe la replicación de varios virus de ADN y ARN, incluida la mayoría de los coronavirus humanos. Recientemente, se descubrió que la cloroquina inhibe el SARS CoV-2 in vitro y su forma hidroxilada se ha propuesto como una posible terapia para tratar pacientes infectados con SARS$\mathrm{CoV}-2^{24}$.

De 20 pacientes hospitalizados mayores de 12 años con COVID-19 confirmado por PCR en muestra nasofaríngea tratados con hidroxicloroquina, seis pacientes recibieron azitromicina (500 mg en el día 1 seguido de $250 \mathrm{mg}$ por día, los siguientes cuatro

Revista Methodo: Investigación Aplicada a las Ciencias Biológicas. Universidad Católica de Córdoba. Jacinto 
días) para prevenir la sobreinfección bacteriana bajo el control diario del electrocardiograma. Al comparar el efecto del tratamiento con hidroxicloroquina como un fármaco único y el efecto de la hidroxicloroquina y azitromicina en combinación, la proporción de pacientes que tuvieron resultados negativos de PCR en muestras nasofaríngeas fue significativamente diferente entre los dos grupos en los días 3-4-5 y 6 postinclusión. En el día 6 posterior a la inclusión, el $100 \%$ de los pacientes tratados con la combinación de hidroxicloroquina y azitromicina se curaron virológicamente en comparación con el $57.1 \%$ en pacientes tratados con hidroxicloroquina solamente, y el $12.5 \%$ en el grupo control ( $p$ $<0.001)$. Por lo tanto, recomiendan que los pacientes con COVID-19 sean tratados con hidroxicloroquina y azitromicina para curar su infección y limitar la transmisión del virus a otras personas para frenar la propagación de COVID$19^{25}$.

\section{Suero convaleciente}

El suero convaleciente humano es una opción para la prevención y el tratamiento de la enfermedad COVID-19 que podría estar disponible rápidamente cuando haya un número suficiente de personas que se hayan recuperado y puedan donar suero que contenga inmunoglobulina. La terapia pasiva implica la administración de anticuerpos a un individuo susceptible con el propósito de prevenir o tratar una enfermedad infecciosa de cierto agente. Por el contrario, la vacunación activa requiere la inducción de una respuesta inmune que tarda en desarrollarse y varía según el receptor de la vacuna. Por lo tanto, la administración pasiva de anticuerpos es el único medio de proporcionar inmunidad inmediata a personas susceptibles. En el caso del SARS-CoV-2, el mecanismo de acción anticipado por el cual la terapia pasiva de anticuerpos mediaría la protección es la neutralización viral. Sin embargo, la desventaja es la citotoxicidad celular dependiente de anticuerpos y / o la fagocitosis ${ }^{26,27}$.

Aunque muchos tipos de preparaciones están o estarán pronto en desarrollo, el único tipo de anticuerpo que está disponible actualmente para uso inmediato es el que se encuentra en los sueros convalecientes humanos. A medida que más personas contraen COVID-19 y se recuperen, el número de donantes potenciales continuará aumentando ${ }^{26}$.

Para desplegar la administración de suero convaleciente para COVID-19 se deben cumplir las siguientes seis condiciones: (i) disponibilidad de una población de donantes que se hayan recuperado de la enfermedad y puedan donar suero convaleciente; (ii) instalaciones de bancos de sangre para procesar las donaciones de suero; (iii) disponibilidad de ensayos, incluidos ensayos serológicos, para detectar SARS-CoV-2 en suero y ensayos virológicos para medir la neutralización viral; (iv) apoyo de laboratorio de virología para realizar estos ensayos; (v) profilaxis y protocolos terapéuticos, que deben incluir ensayos clínicos aleatorios para evaluar la eficacia de cualquier intervención y medir las respuestas inmunes; y (vi) cumplimiento normativo, incluida la aprobación de la junta de revisión institucional, que puede variar según la ubicación ${ }^{26}$.

El 28 de febrero de 2020, la Comisión Nacional de Salud de la República Popular China (NHCPRC) anunció que de los 157 sujetos con COVID-19 gravemente enfermos transfundidos con plasma procesado recolectado de pacientes rehabilitados, 91 mostraron una mejora significativa dentro de las 48 horas de tratamiento. Mejoras que incluían aumento en la proporción de linfocitos, saturación de oxígeno en la sangre y reducción en el nivel de antígeno viral. Además, hubo una mejora sintomática significativa, como reducción y ausencia de fiebre, tos, esputo, dolor muscular y debilidad. El NHCPRC declaró que la terapia con plasma era segura y eficaz. China había recolectado 544 muestras de plasma de donantes voluntarios rehabilitados para tratar 245 casos de pacientes críticos ${ }^{28}$.

\section{Vacuna}

Existen varias formas de producción de vacunas que están siendo aplicadas para la producción de una funcional contra el SARS-CoV-2. Las vacunas de virus enteros atenuados o inactivos representan una estrategia clásica para las vacunas virales. Johnson \& Johnson es una de las compañías multinacionales que está empleando el vector adenovírico AdVac ${ }^{\circledR}$ de Janssen y fabricando en su tecnología de línea celular PER.C6®, por otro lado, los investigadores de la Universidad de Hong Kong han desarrollado una vacuna viva contra la influenza que expresa las proteínas del SARSCoV-2. Finalmente, Codagenix ha desarrollado una tecnología de "desoptimización de codones" para atenuar los virus y está explorando estrategias de vacuna contra el SARS-CoV-2 ${ }^{21}$.

Las vacunas de subunidades para ambos coronavirus del SARS se basan en provocar una respuesta inmune contra la proteína espiga $S$ para evitar su acoplamiento con el receptor ACE2 del huésped. La Universidad de Queensland está sintetizando proteínas virales de la superficie, para presentarlas más fácilmente al sistema inmunitario. Además, Novavax ha desarrollado y producido nanopartículas inmunogénicas similares a virus basadas en la expresión recombinante de la proteína $\mathrm{S}$, mientras que Clover

Revista Methodo: Investigación Aplicada a las Ciencias Biológicas. Universidad Católica de Córdoba. Jacinto 
Biopharmaceuticals está desarrollando una vacuna subunitaria que consiste en una proteína S SARSCoV-2 trimerizada utilizando su tecnología patentada Trimer-Tag ${ }^{\circledR}$, aunque algunas proteínas $\mathrm{S}$ de longitud completa para el SARS también provocan una mayor infectividad e infiltración eosinófila. En consecuencia, un consorcio liderado por el Centro de Desarrollo de Vacunas del Texas Children's Hospital en el Baylor College of Medicine (incluyendo la Rama Médica de la Universidad de Texas y el Centro de Sangre de Nueva York) ha desarrollado y probado una vacuna subunitaria compuesta solo por el dominio de unión al receptor (RBD) de la proteína $S$ del SARS-CoV. Cuando se formula en alumbre, la vacuna SARSCoV RBD provoca altos niveles de inmunidad protectora en el desafío del virus homólogo. Una ventaja de la vacuna basada en RBD es su capacidad para minimizar la inmunopotenciación del huésped. Los hallazgos iniciales de que los SARS-CoV y SARS-CoV-2 muestran más del $80 \%$ de similitud de aminoácidos y se unen al mismo receptor ACE2 ofrecen la oportunidad de desarrollar cualquiera de las proteínas como una vacuna subunitaria ${ }^{21}$.

Inovio Pharmaceuticals está desarrollando una vacuna de ADN, mientras que otras, como Moderna Therapeutics y Curevac, están explorando plataformas de vacunas de ARN. El concepto de inmunización con ADN comenzó con resultados prometedores en ratones en 1993 que mostraron inmunidad protectora contra la gripe, pero durante décadas, estos hallazgos no se han traducido en hallazgos similares en humanos. Más recientemente, las nuevas modificaciones y formulaciones han mejorado el rendimiento del ácido nucleico en humanos, con la expectativa de que este enfoque podría conducir a la primera vacuna con licencia de ácido nucleico humano ${ }^{21,29}$.

\section{Tasa de letalidad y la tasa de letalidad por infección}

Se poseen estadísticas de muchos países sobre nuevas infecciones y muertes causadas por este virus, lo que provoca opiniones diferentes sobre la gravedad y la progresión de la pandemia. Evaluar la gravedad es fundamental para la formulación de políticas $\mathrm{y}$, por lo tanto, se ha puesto mucho esfuerzo en la cuantificación.

Una medida comúnmente utilizada es la tasa de letalidad (CFR), que es la proporción de muertes del total de casos diagnosticados. El CFR reportado en diferentes países varía significativamente, va desde $0.3 \%$ al $10 \%$. Varios factores clave afectan el CFR, como parámetros demográficos y prácticas asociadas con un mayor o menor riesgo difieren mucho entre las sociedade ${ }^{30}$. Generalmente hay un retraso entre la aparición de los síntomas y la muerte, lo que puede conducir a una subestimación de la CFR al comienzo de la progresión de una epidemia. Por lo tanto, el CFR tenderá a sobreestimar la tasa de muertes por persona infectada, denominada tasa de mortalidad por infección (IFR) ${ }^{31}$.

La estimación del número total de personas infectadas generalmente se logra mediante la prueba de una muestra aleatoria de anticuerpos antivirales, cuya presencia indica que el paciente estaba previamente infectado. Al momento de escribir, tales ensayos no están ampliamente disponibles, por lo que los investigadores recurren a conjuntos de datos sustitutos generados por las pruebas de ciudadanos extranjeros que regresan a casa de países infectados, o modelos epidemiológicos que estiman el número de casos indocumentados. Estos métodos proporcionan una primera visión de la verdadera gravedad de la enfermedad $^{31}$.

Un estudio realizado para obtener estimaciones ajustadas y específicas por edad de la tasa de letalidad general (CFR) de COVID-19 entre pacientes sintomáticos y asintomáticos en la provincia de Hubei, entre el 1 de enero y el 11 de febrero, mostró que 1.6\% (1.4-1.8) de las personas infectadas con COVID-19 durante ese período con o sin síntomas murieron o morirán, con diferencias aún más importantes por grupo de edad que lo sugerido por los datos sin procesar. La probabilidad de muerte entre las personas infectadas con síntomas se estima en 3.3\% (2.93.8 ), con un fuerte aumento en el grupo de mayores de 65 años y del $36 \%$ en mayores de 80 años $^{32}$.

\section{Mortalidad}

Una compilación de estudios epidemiológicos mostró que la edad media de 425 pacientes reportados en el epicentro del brote (Wuhan en la provincia de Hubei, China) fue de 59 años, se reportó mayor morbilidad y mortalidad entre los ancianos y entre aquellos con afecciones coexistentes (similar a la situación con influenza); el $56 \%$ de los pacientes eran varones. No hubo casos en niños menores de 15 años porque tienen menos probabilidades de infectarse o sus síntomas fueron tan leves que su infección escapó a la detección, lo que tiene implicaciones para el tamaño del denominador de las infecciones comunitarias totales ${ }^{15,33}$.

En otra investigación, entre 1099 pacientes con Covid-19 confirmado por laboratorio, la mortalidad fue del 1,4\%,34 lo que sugiere que las consecuencias clínicas generales de Covid-19 pueden ser más parecidas a las de un influenza estacional severa (que tiene una tasa de letalidad de aproximadamente $0.1 \%$ ) o influenza pandémica (similar a las de 1957 y 1968) en lugar de una

Revista Methodo: Investigación Aplicada a las Ciencias Biológicas. Universidad Católica de Córdoba. Jacinto 
enfermedad similar al SARS o MERS, que han tenido tasas de letalidad de 9 a $10 \%$ y $36 \%$, respectivamente ${ }^{35}$.

\section{Casos en Argentina}

Con base a los datos oficiales del Ministerio de Salud Argentino se graficó la evolución temporal del número de infectados versus tiempo, medido en días. El resultado mostrado en la (figura 1) corresponde a los 97 días que transcurrieron desde la aparición del primer caso (03 de marzo de 2020) hasta el 09 de junio del 2020. La curva notoriamente creciente ajustó, con factor de correlación de 0.9961 , a un crecimiento exponencial (no mostrado en la figura 1). Este resultado junto con el hecho de que el 9 de junio se registró una tasa de contagio con 1141 nuevos casos, obliga a replantearse tomar medidas más estrictas durante la cuarentena. Desde el día 76 se observó en la curva un aumento de velocidad (19 de mayo de 2020) lo que pudiera coincidir con un relajamiento de la cuarentena, ya que se adoptaron medidas de apertura muy audaces acompañadas de protestas "anti-cuarentena", lo que pudo haber provocado un aceleramiento de $\operatorname{casos}^{36,37}$.

El número de recuperados en el tiempo se muestra en la (figura 2). Los enfermos que son hospitalizados comienzan a recuperarse a los 25 días aproximadamente. El crecimiento de los recuperados aumenta exponencialmente según pudo constatarse con la curva de ajuste que presenta la siguiente ecuación con factor de correlación de 0.9963 .

En Argentina la primera muerte corresponde a un hombre de 64 años, ocurrió al sexto día del primer diagnóstico positivo en Argentina (09/03/20) además de ser el primer deceso por COVID-19 en América Latina. La (figura 3) muestra el número de fallecidos en todo el país en función del tiempo. Para la (figura 4), se discriminó el número de fallecidos por edad y se desglosó los datos considerando intervalos de edades de diez en diez, desde los 40 hasta mayores de 90 años. Los resultados muestran que el mayor número de fallecidos están entre 70 y 89 años y la velocidad de crecimiento de muertes es mayor entre 80 y 89 años.

La (figura 5) muestra el número de muertos por edad hasta el día 97 de la pandemia (09 de junio de 2020), donde se corrobora que la mayor cantidad de muertos están entre 70 y 89 años.

Entre los 0 a 39 años, la incidencia es estadísticamente despreciable, esta se vuelve relevante a partir de los 40 años en adelante. En los reportes del Ministerio de Salud argentino enfatizan que las muertes suceden en su mayoría en presencia de enfermedades preexistentes ${ }^{36}$.
La (figura 6) muestra el número de casos por día. Se nota que, a partir de la aplicación de la cuarentena hasta el día 60 aproximadamente, la velocidad de crecimiento se mantuvo constante, lo que mantuvo la velocidad de crecimiento acotado. Estas

fluctuaciones producen una velocidad promedio de aumento de casos inferior a la velocidad teórica que es exponencial. Sin embargo, a partir del día 60 en adelante se observa un dramático aumento de la cantidad de casos, lo que coincide con una relajación del rigor de la cuarentena; debido a que a mediados de abril el Gobierno argentino autoriza la apertura de bancos y de otros establecimientos que desarrollen actividades de cobranza de servicios e impuestos, además de habilitar la atención médica y odontológica con cita previa, laboratorios de análisis clínicos y centros de diagnóstico por imagen y en ópticas; haciendo que más personas salgan a las calles ${ }^{38}$.

\section{Medidas de Prevención}

En ausencia de cualquier intervención farmacéutica, la única estrategia contra COVID-19 es reducir el contacto entre personas sanas e infecciosas. Para esto se recomienda procesos de intervención combinada, desde la detección temprana de casos hasta la aplicación de cuarentena, es decir, el distanciamiento social. La intervención combinada redujo el número medio estimado de infecciones en un $99.3 \%$ (92.6-99.9) cuando R0 era 1.5 , en $93.0 \%$ (81.5-99.7) cuando R0 era 2.0, y en 78.2\% (59.0-94.4) cuando R0 era 2.5. La observación de que la mayor reducción en los casos de COVID-19 se logró bajo la intervención combinada no es sorprendente ${ }^{39}$.

De igual manera, la Organización Mundial de la Salud recomienda que las personas usen máscaras faciales, para evitar una posible transmisión asintomática o presintomática ${ }^{40}$. Se demostró la eficacia de las máscaras quirúrgicas para reducir la detección de coronavirus y las copias virales en gotas respiratorias grandes y en aerosoles. Esto tiene implicaciones importantes para el control de COVID-19, lo que sugiere que las personas enfermas podrían usar máscaras faciales quirúrgicas para reducir la transmisión posterior ${ }^{41}$.

\section{Conclusiones}

Este artículo presenta una recopilación de lo que se conoce hasta la fecha sobre el virus SARS-CoV-2 y la enfermedad que causa: COVID-19. De la revisión realizada se resalta el origen natural del mismo y el proceso de infección, destacando la proteína espiga (S) que se encuentra en la 
superficie del virus, la cual se acopla al receptor ACE2 presente en varios tipos celulares de los humanos. Debido a esta característica, la COVID19 muestra una gran variedad de síntomas, desde afecciones al sistema pulmonar hasta problemas del sistema digestivo.

Al ser un virus nuevo, los métodos de diagnóstico y tratamiento aun no son $100 \%$ eficientes, pero varios laboratorios alrededor del mundo están trabajando para poder prevenir la enfermedad mediante una vacuna, tratar a las personas infectadas y mejorar los métodos de diagnóstico para poder reducir la tasa de mortalidad.

Una cuestión clave para los epidemiólogos es ayudar a los encargados de formular políticas a decidir los objetivos principales de la mitigación, por ejemplo, minimizar la morbilidad y la mortalidad asociada, evitar un pico epidémico que sobrepase los servicios de atención de la salud, mantener los efectos en la economía dentro de niveles manejables y aplastar la curva epidémica. Como se observan en Argentina y en el mundo, aún queda muchos vacíos que llenar sobre información de este virus, los casos de esta enfermedad siguen en aumento, por lo que las medidas de prevención son clave mientras no se posea una solución farmacológica. Para COVID19, el distanciamiento social (detención de las reuniones masivas, cierre de institutos educativos o lugares de trabajo y el aislamiento de las personas infectadas) ha demostrado que reducen el valor del número efectivo de reproducción $\mathrm{R}$. Si a esto se le suma el uso de mascarillas, como recomienda la Organización Mundial de la Salud y los Ministerios de Salud Pública de cada país, se podría manejar de manera adecuada la enfermedad sin la necesidad de que la economía del país se vea gravemente afectada ${ }^{37}$.

\section{Bibliografía}

1. Huang, C., Wang, Y., Li, X., et al. Clinical features of patients infected with 2019 novel coronavirus in Wuhan, China. Lancet. 2020; 395(10223): 497-506. https://doi.org/10.1016/S01406736(20)30183-5.

2. Zhao, D., Yao, F., Wang, L., et al. A comparative study on the clinical features of COVID-19 pneumonia to other pneumonias.

$$
\text { J. Infect. Dis. 2020; } 21 .
$$
https://doi.org/10.1093/cid/ciaa247.

3. Corman, V. M., Muth, D., Niemeyer, D., \& Drosten, C. Hosts and Sources of Endemic Human Coronaviruses. Adv. Virus Res. 2018; Vol. $100, \quad$ pp.
https://doi.org/10.1016/bs.aivir.2018.01.001

4. Jiang, F., Deng, L., Zhang, L., YinCai, Cheung, C. W., \& Xia, Z. Review of the Clinical Characteristics of Coronavirus Disease 2019 (COVID-19). J Gen Intern Med. 2020 1-5. DOI: 10.1007/s11606-020-05762W.

5. Andersen, K. G., Rambaut, A., Lipkin, W. I., Holmes, E. C., \& Garry, R. F. The proximal origin of SARS-CoV-2. Nat. Med. 2020. https://doi.org/10.1038/s41591-020-0820-9.

6. Walls, A. C., Park, Y. J., Tortorici, M. A., Wall, A., McGuire, A. T., \& Veesler, D. Structure, Function, and Antigenicity of the SARS-CoV-2 Spike Glycoprotein. Cell. 2020. https://doi.org/10.1016/j.cell.2020.02.058.

7. Wan, Y. Molecular mechanism for antibodydependent enhancement of coronavirus. J. Virol. 2020; 94(5):e02015-19. https://doi.org/10.1164/rccm.200702-2710C.

8. Wan, Y., Shang, J., Graham, R., Baric, R. S., \& Li, F. Receptor Recognition by the Novel Coronavirus from Wuhan: an Analysis Based on Decade-Long Structural Studies of SARS Coronavirus. J. Virol. 2020; 94(7). https://doi.org/10.1128/jvi.00127-20.

9. Wrapp, D., Wang, N., Corbett, K. S., et al. Cryo-EM structure of the 2019-nCoV spike in the prefusion conformation. Science. 2020; 367(6483):

1260-1263. https://doi.org/10.1126/science.aax0902.

10. Zhang, J., Ma, X., Yu, F., Liu, J., Zou, F., \& Pan, T. Teicoplanin potently blocks the cell. BioRxiv. 2020; 1-7. (DOI: https://doi.org/10.1101/2020.02.05.935387.)

11. Zhang, C., Shi, L., \& Wang, F.-S. Liver injury in COVID-19: management and challenges. Lancet Gastroenterol. Hepatol. 2020; 1-3. DOI: https://doi.org/10.1016/S24681253(20)30057-1.

12. Zhang, T., Wu, Q., \& Zhang, Z. Pangolin homology associated with 2019-nCoV. BioRxiv. 2020; 950253. https://doi.org/10.1101/2020.02.19.950253.

13. Zhang, T., Wu, Q., \& Zhang, Z. Probable Pangolin Origin of SARS-CoV-2 Associated with the COVID-19 Outbreak. Curr. Biol. 2020. doi: 10.1016/j.cub.2020.03.022.

14. Lirong Zou. SARS-CoV-2 Viral Load in Upper Respiratory Specimens of Infected Patients. N. Engl. J. Med. 2020; 1-3. DOI: 10.1056/NEJMc2001737. 
15. Fauci, A., Lane, C., \& Redfield, R. R. Covid19 - Navigating the Uncharted. N. Engl. J. Med. 2020;1-2. DOI: 10.1056/NEJMe2002387.

16. Holshue, M., DeBolt, C., Lindquist, S., et al. First Case of 2019 Novel Coronavirus in the United States. N. Engl. J. Med. 2020; 1-9. DOI: 10.1056/NEJMoa2001191.

17. Lauer, S.-A., Grantz, K.-H., Bi, Q., et al. The Incubation Period of Coronavirus Disease 2019 (COVID-19) From Publicly Reported Confirmed Cases: Estimation and Application. Ann. Intern. Med. 2020. doi:10.7326/M20-0504.

18. Koh, G. C.-H., \& Hoenig, H. How Should the Rehabilitation Community Prepare for 2019nCoV? Arch Phys Med Rehabil. 2020. doi: 10.1016/j.apmr.2020.03.003.

19. Wang J, Wang BJ, Yang JC, et al. Advances in the research of mechanism of pulmonary fibrosis induced by Corona Virus Disease 2019 and the corresponding therapeutic measures. Zhonghua Shao Shang Za Zhi. 2020. DOI: $\quad 10.3760 /$ cma.j.cn50112020200307-00132.

20. Rismanbaf, A., \& Zarei, S. Liver and Kidney Injuries in COVID-19 and Their Effects on Drug Therapy; a Letter to Editor. Acad Emerg Med. 2020; 8(1): $\quad$ e17. http://journals.sbmu.ac.ir/aaem.

21. Cheng, Y., Luo, R., Wang, K., Zhang, M., Wang, Z., \& Dong, L. Kidney impairment is associated with in-hospital death of COVID19 patients. MedRxiv. 2020; 1-21. DOI: https://doi.org/10.1101/2020.02.18.20023242

22. Ungaro, R. C., Sullivan, T., Colombel, J.-F., \& Patel, G. What Should Gastroenterologists and Patients Know About COVID-19? Clin. Gastroenterol. Hepatol. 2020; 1-12. doi: 10.1016/j.cgh.2020.03.020.

23. Touret, F., \& de-Lamballerie, X. Of chloroquine and COVID-19. Antiviral Res. 2020; 177: 1-2. (doi.org/10.1016/j.antiviral.2020.104762).

24. Baron, Devaux, C., Colson, P., D., \& Rolain, J.-M. Teicoplanin: an alternative drug for the treatment of coronavirus COVID-19? Int. J. Antimicrob. Agents. 2020; 1-7. doi: 10.1016/j.ijantimicag.2020.105944.

25. Gautret, P., Lagier, J.-C., Parola, P., Hoang, V.-T., Meddeb, L., \& Mailhe, M. Hydroxychloroquine and azithromycin as a treatment of COVID-19: results of an open- label non-randomized clinical trial. Int. J. Antimicrob. $\quad 2020$. https://doi.org/10.1016/j.ijantimicag.2020.10 5949.

26. Casadevall, A., \& Pirofski, L.-a. The convalescent sera option for containing COVID-19. J Clin Invest. 2020. https://doi.org/10.1172/JCI138003.

27. Luke, T., Casadevall, A., Watowich, S., Hoffman, S., Beigel, J., \& Burgess, T. Hark back: passive immunotherapy for influenza and other serious infections. Crit Care Med. 2010; 38(4 suppl): e66-e73.

28. Law, P. Emergent Serum Therapy and Antibody Medicine to Counteract Sudden Attacks of COVID-19 and Other Pathogenic Epidemics. Regen. Med. 2020; 9, 1-7. doi: 10.4236/ojrm.2020.91001.

29. Chen, W.-H., Strych, U., Hotez, P., \& Bottazzi, M.-E. The SARS-CoV-2 Vaccine Pipeline: An Overview. Curr. Trop. Med. Rep. 2020; 1-4. https://doi.org/10.1007/s40475020-00201-6.

30. China CDC 2020. Vital Surveillances: The Epidemiological Characteristics of an Outbreak of 2019 Novel Coronavirus Diseases (COVID-19). China CDC Weekly. 2020; 2(8): 113-122.

31. Yinon M. Bar-On, Avi Flamholz, Rob Phillips \& Ron Milo. SARS-CoV-2 (COVID-19) by the numbers. Elife. 2020. Search in: https://elifesciences.org/articles/57309.

32. Riou, J., Counotte, M., Hauser, A., \& Althaus, C. Adjusted age-specific case fatality ratio duringcovid-19 epidemic in Hubei, China, January and February 2020. MedRxiv. 2020; 1-10. DOI: https://doi.org/10.1101/2020.03.04.20031104

33. Li, Q., Guan, X., \& Wu, P. Early transmission dynamics in Wuhan, China, of novel coronavirus-infected pneumonia. N Engl J Med. 2020. DOI: 10.1056/NEJMoa2001316.

34. Guan, W., Ni, Z., \& Hu, Y. Clinical characteristics of coronavirus disease 2019 in China. N Engl J Med. 2020. DOI: 10.1056/NEJMoa2002032.

35. de-Wit, E., van-Doremalen, N., Falzarano, D., \& Munster, V. SARS and MERS: recent insights into emerging coronaviruses. Nat. Rev. Microbiol. 2016; 14:523-534.

36. Ministerio de Salud Argentino [Internet]. Argentina: Ministerio de Salud; 2020 [acceso

Revista Methodo: Investigación Aplicada a las Ciencias Biológicas. Universidad Católica de Córdoba. Jacinto 
09 de junio de 2020]. Nuevo coronavirus COVID-19, Informes diarios. Disponible en: https://www.argentina.gob.ar/coronavirus/inf orme-diario.

37. La Nación [Internet]. Argentina: Rodríguez Niell, P.; 2020. [actualizado 28 de mayo de 2020]. Protestas anticuarentena: la provincia de Buenos Aires y la Ciudad dicen que solo actuarán si lo ordenan los jueces. Disponible en:

https://www.lanacion.com.ar/politica/manifes taciones-anticuarentena-provincia-buenosaires-ciudad-dicen-nid2370648.

38. Telesur [Internet]. Argentina: teleSUR - SH; 2020 [actualizado 19 de abril de 2020]. Argentina relaja cuarentena y exceptúa nuevas actividades- Disponible en: https://www.telesurtv.net/news/argentinarelaja-cuarentena-permitira-actividadescomercia les-20200419-0009.html
39. Lewnard, J. A., \& Lo, N. C. Scientific and ethical basis for social-distancing interventions against COVID-19. Lancet Infect. Dis. 2020. doi:10.1016/s14733099(20)30190-0.

40. Feng, S., Shen, C., Xia, N., Song, W., Fan, M., Cowling, B. Rational use of face masks in the COVID-19 pandemic. Lancet. 2020. DOI: https://doi.org/10.1016/S22132600(20)30134-X

41. Leung, N., Chu, D., Shiu, E., et al. Respiratory virus shedding in exhaled breath and efficacy of face masks. Nat. Med. 2020. DOI: https://www.nature.com/articles/s41591-020$\underline{0843-2}$

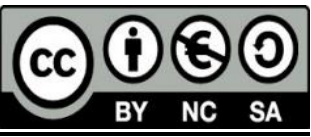

\section{Anexo figuras}

- Con datos oficiales del Ministerio de Salud Argentino se graficó la evolución del número de infectados, recuperados y de muertos por edad versus tiempo.

- Se indica el origen, mecanismo de infección, síntomas, secuelas, diagnóstico, posible tratamiento y medidas de atenuación.

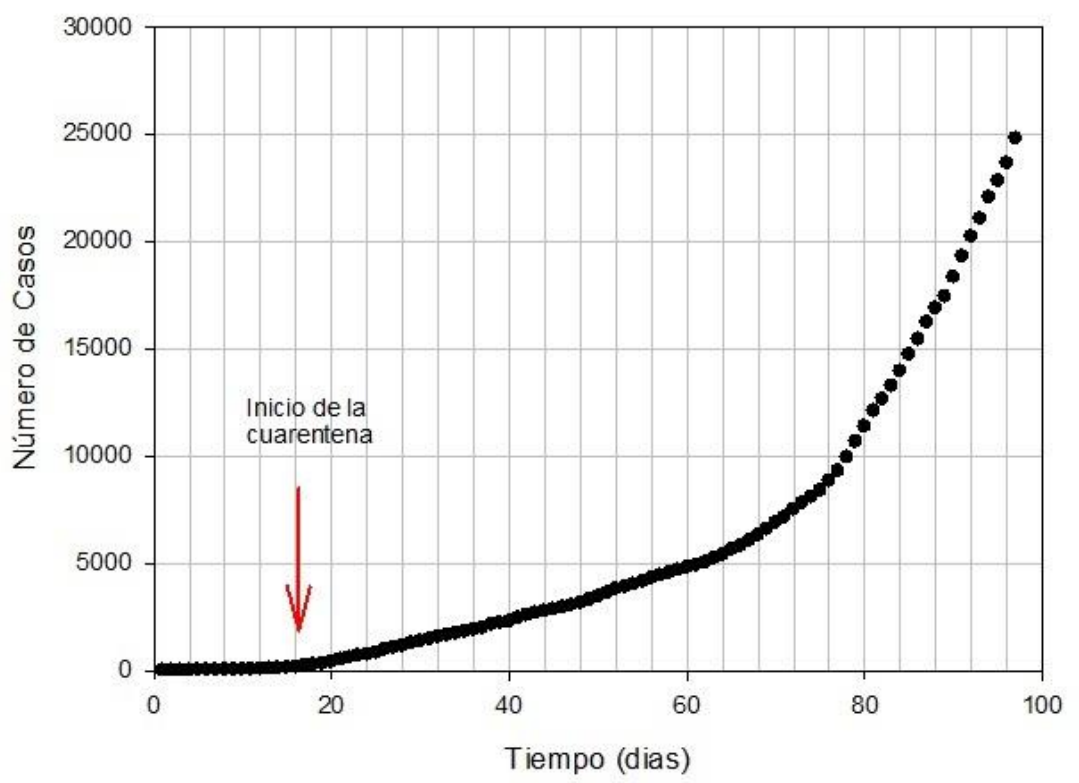

Figura 1. Número de infectados versus tiempo. El crecimiento de esta curva ajusta a una función exponencial. 
Terán T, Layedra A, Martinez L A, Cadena M F, Pérez S M, Lerner B. Sars-cov-2 y sus efectos en el primer trimestre en Argentina.

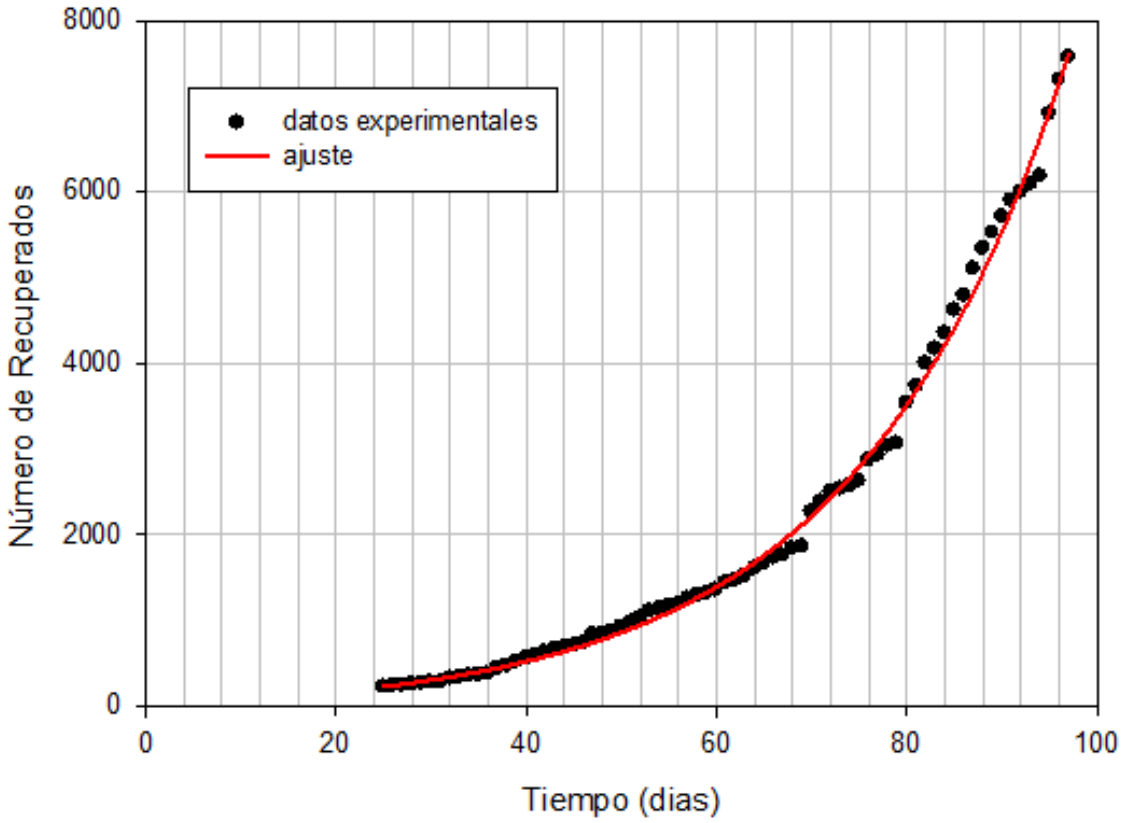

Figura 2. Número de recuperados versus tiempo junto con el ajuste de los datos y que obedece una función exponencial.

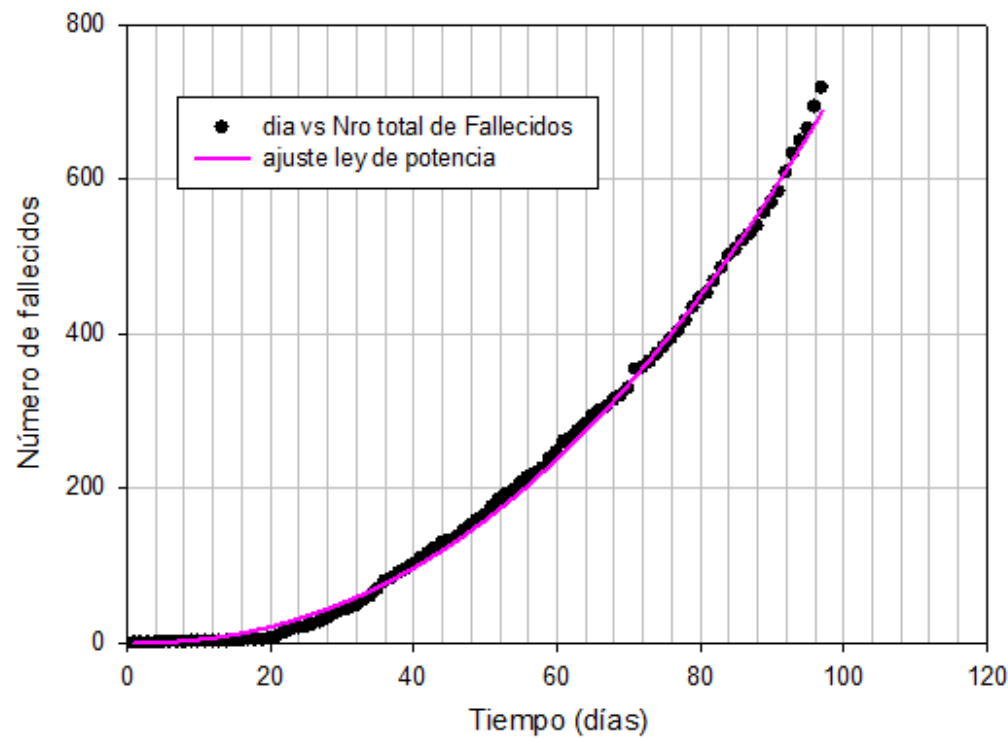

Figura 3. Número de Fallecidos en todo el país versus día. 


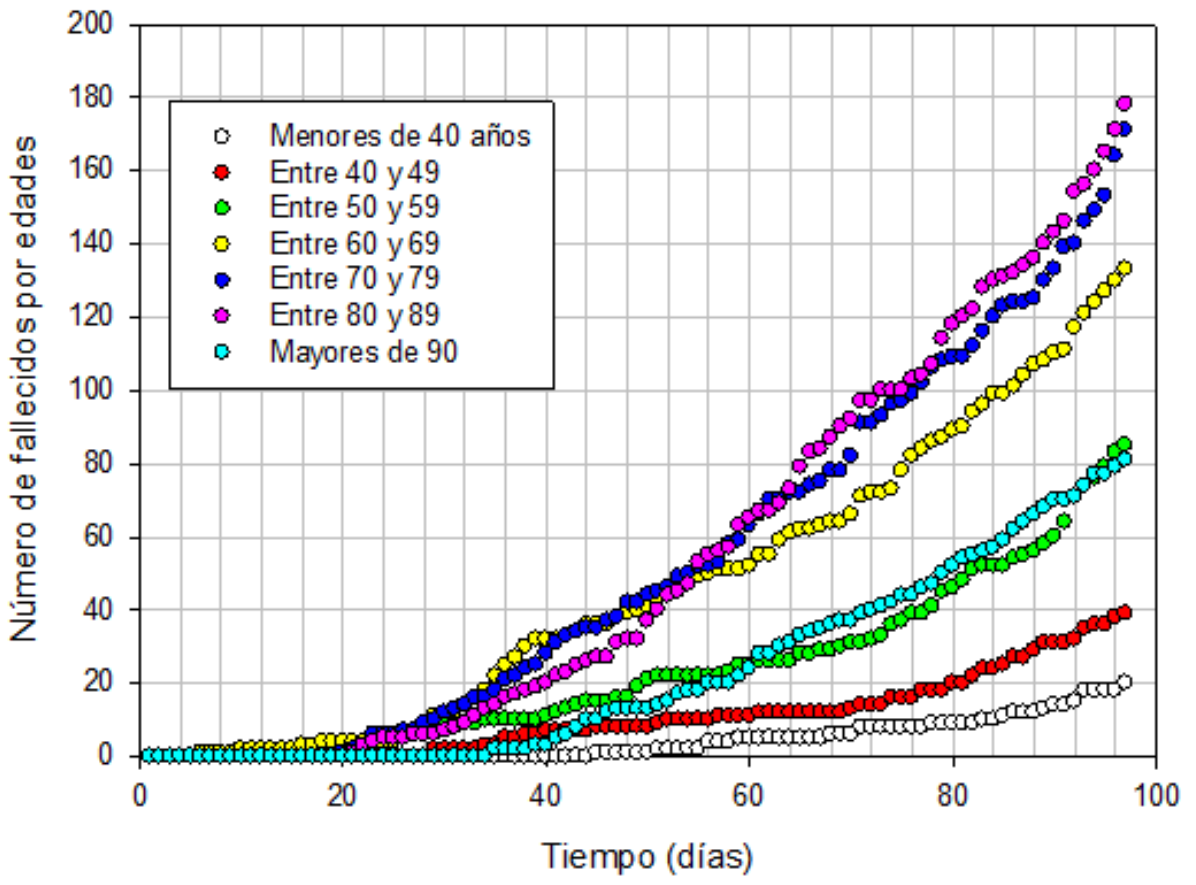

Figura 4. Número de fallecidos por edades versus tiempo.

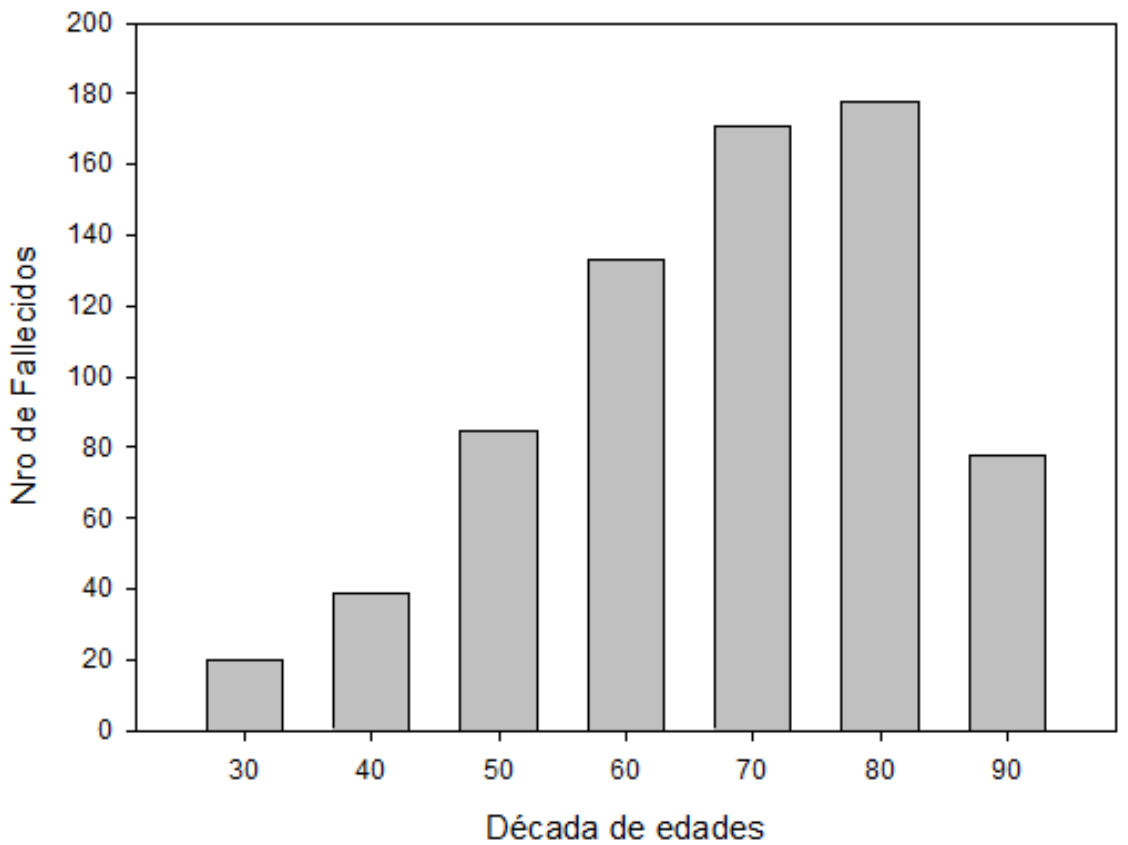

Figura 5. Gráfico de Barras del número de fallecidos por edad para el día 97 (09 de junio de 2020). 
Terán T, Layedra A, Martinez L A, Cadena M F, Pérez S M, Lerner B. Sars-cov-2 y sus efectos en el primer trimestre en Argentina.

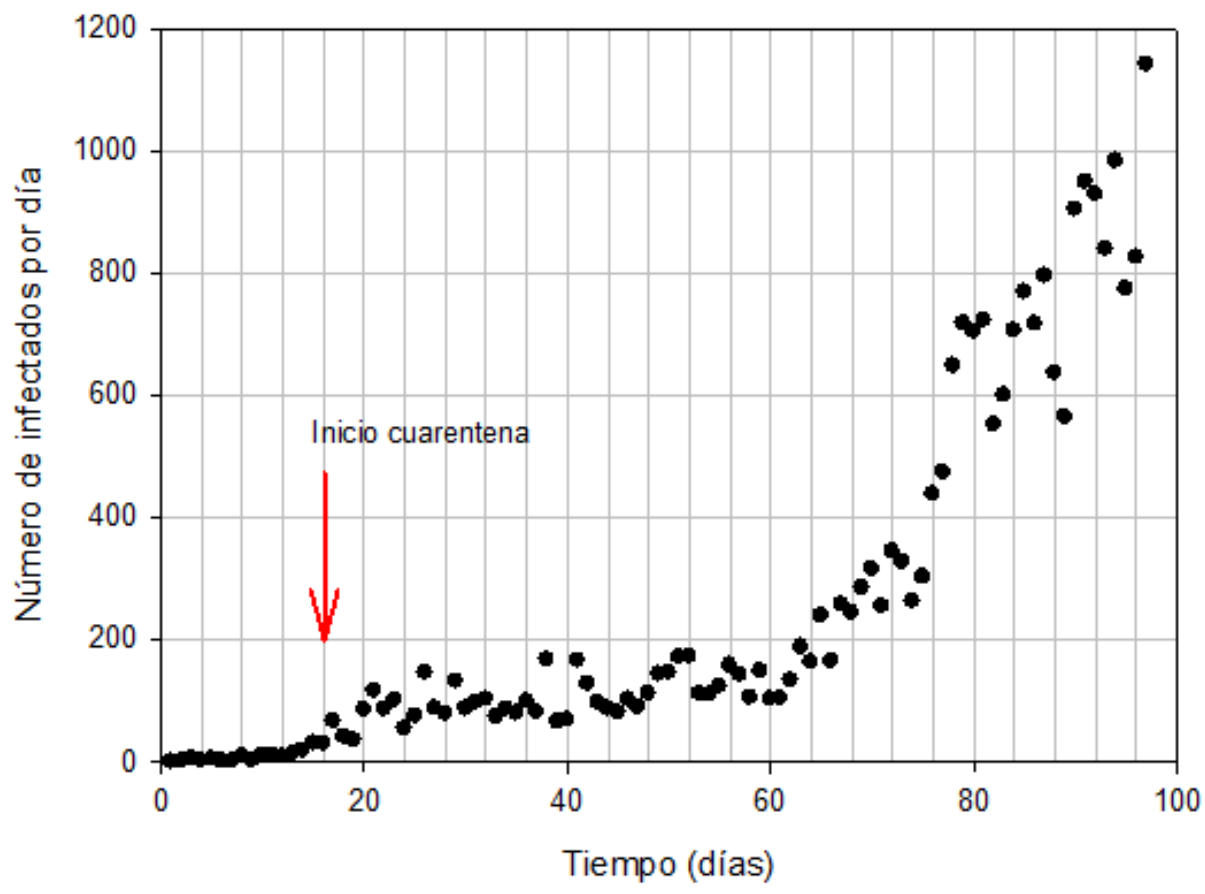

Figura 6. Número de infectados por día vs tiempo.

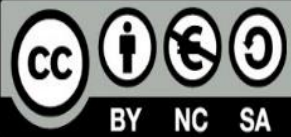

Revista Methodo: Investigación Aplicada a las Ciencias Biológicas. Universidad Católica de Córdoba. Jacinto Ríos 571 Bo Gral. Paz. X5004FXS. Córdoba. Argentina. Tel.: (54) 3514517299 / Correo: methodo@ucc.edu.ar / Web: methodo.ucc.edu.ar | ARTICULO DE REVISION Rev. Methodo 2021;6(1):20-32. 